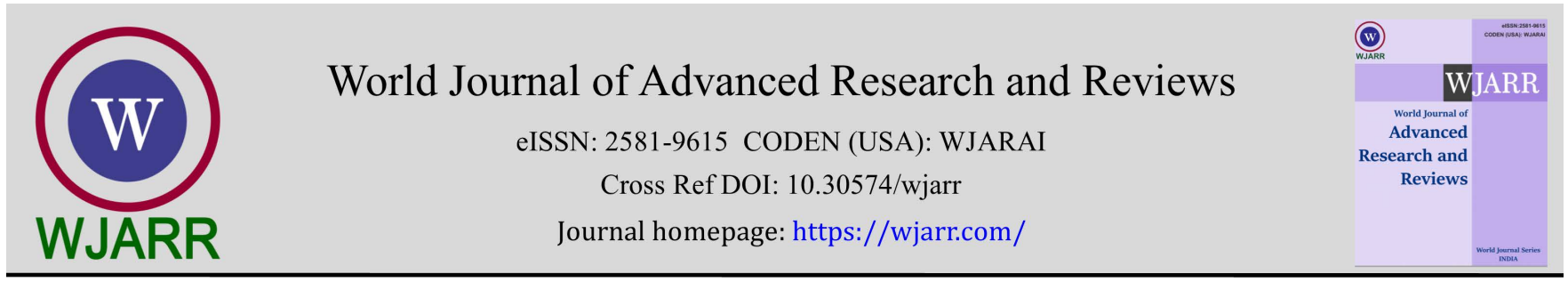

(REVIEW ARTICLE)

\title{
A comparative study of Psychological analysis during intermaxillary fixation between second and third decade
}

\author{
MC Prasant Pillai, Kapil Lahoti, Pavan D Tenglikar, Aakanksha Sharma * and Shiv Pratap Singh \\ Department of oral and maxillofacial surgery RKDF dental college and research centre Bhopal.
}

World Journal of Advanced Research and Reviews, 2022, 13(01), 689-692

Publication history: Received on 12December 2021; revised on 22 January 2022; accepted on 24 January 2022

Article DOI: https://doi.org/10.30574/wjarr.2022.13.1.0058

\begin{abstract}
To access the impact of stress during imf by doing psychological analysis of the two-age group as imf has huge impact on the patient. The purpose of this prospective study was to evaluate levels of depression, anxiety, and stress, at three time points using DASS-21 (Depression, Anxiety and Stress Scale-21). The two age groups were taken mid twenty and mid thirty both groups were managed by intermaxillary fixation. the comparative study of psychological analysis was done by using DASS-21 SCALE, the questionnaires' were distributed to rate the analysis for depression, anxiety and stress. Scores for depression and stress rose significantly in the first group of mid-twenties compared to mid-thirties. The DASS-21 was a useful method of evaluating stress. Correlation of the results from the two questionnaires showed a strongly positive association.
\end{abstract}

Keywords: Intermaxillary fixation; Stress; Psychological analysis; Fracture; Weightloss

\section{Introduction}

Although intermaxillary fixation (IMF) is performed to treat the patients with maxillary fracture, this procedure is very stressful to the patients. IMF has been reported to increase noradrenaline (NA) release in the brain and elevate plasma corticosterone, during fracture or any breakage in the bone it can be fixed by application by plaster bandage by close reduction but in oral cavity it cannot be done, to fix fractures of oral cavity procedure of close reduction Is done in oral cavity as intermaxillary fixation is one of the most commonly used treatment protocol for mid face fracture [1].

When treated and imf is done, patients experience several physical, mental, emotional, and social difficulties, and the effects of the treatment on daily activities such as chewing, swallowing, and speech, can cause depression, anxiety, and stress, which must be treated to enable patients to cope [2].

Many studies have reported the difficulties patients face, weight loss and several have focused on anxiety and depression, but to our knowledge few have looked at potential anxiety or stress. Patients who are stressed typically find it difficult to relax, and are irritable, tense, and agitated. Various analytical studies have reported a correlation among stress, depression, and anxiety,but few if any data about all three concurrently. The purpose of this study there for eis to evaluate depression, anxiety, and stress in patients with intermaxillary fixation Questionnaire showed that most patients noted insomnia and an irritated feeling during IM [1].

The purpose of this study therefore was to evaluate depression, anxiety, and stress in patients with oral cancer using the short version of the Depression Anxiety and Stress Scale (DASS-21[2].

\footnotetext{
${ }^{*}$ Corresponding author: Aakanksha Sharma

Department of oral and maxillofacial surgery RKDF dental college and research centre Bhopal.

Copyright $(2022$ Author(s) retain the copyright of this article. This article is published under the terms of the Creative Commons Attribution Liscense 4.0.
} 


\section{Psychological stress and stress-activated pathways}

Stress as a specific medical term was first defined by the endocrinologist Hans Selye in 1936as the physiological adaptive responses to perceived (psychological) or real (physical) threats ("stressors") to an organism. An acute (sudden or short-term) stressor can evoke a "fight or flight" response that prepares to defend the stability of the internal environment in order to ensure the survival of the organism. When the stress passes, negative feedback is triggered to terminate the stress response and bring the body back to a state of homeostasis or eustasis.

However, if the stressor becomes chronic and/ or exceeds the organism's ability to maintain the stress response, it becomes harmful because basal homeostasis cannot be reached. For most humans in modern societies, psychological stress is more frequent than physical stress and it may be induced by various social and emotional triggers, some of which can be unique for an individuals[3].

\section{Patients and methods}

The cases werestudied who were treated at the Department of Oral and Maxillofacial Surgery at RKDF Dental college and research center, Bhopal Those with fracture, who were 18 years and above,the patient in the institute were categorized in two groups where first group is in mid-twenties and second group is in mid-thirties and able to read and write in English or Hindi, were included. Those who had a history of mental illness,difficulty in communication or had psychological treatment for a mental illness, were excluded. Out of 51 patients who were enrolled for the study 11 refused and so 40 cases were included in the study.They each completed the DASS-21 and HADS at three different time points during their treatment.

\section{Questioners}

Questionnaires DASS-21 (Depression, Anxiety, and Stress Scale) To evaluate depression, anxiety, and stress, we used the DASS-21, a shorter version of the DASS-42, in both the Hindi and English languages. Each of the three domains includes seven items, which are scored from 0 (does not apply to me at all) to 3 (applies to me very much, or most of the time). Scores for each domain are added to give the total. We chose this questionnaire as it measures depression, anxiety, and stress at the same time, and does not take into account cultural background, and we chose the short version to save time. The domain "depression" evaluates low self-esteem, low positivity, and hopelessness.

The anxiety domain assesses subjective feelings of fear, physiological hyperarousal, and autonomic arousal, and the stress domain assesses negativity, agitation, and tension. This is in accordance with the tripartite theory, which suggests that anhedonia (loss of pleasure), or lack of positivity, is an integral factor that differentiates depression from anxiety. High scores for depression, anxiety, and stress, are characterised as .mood disorder, panic disorder, and general anxiety disorder, respectively, according to the Diagnostic and Statistical Manual of Mental Disorders edition 4 (DSM-IV).(4)

\section{Statistical analysis}

HADS (Hospital Anxiety and Depression Scale

This scale comprises two domains: depression and anxiety, each with seven questions that are scored on a Likert scale (0-3). Total scores for each domain are calculated separately.Statistical analysis Data were .analysed with the help of IBM SPSS Statistics for Windows, version 20 (IBM Corp). Probabilities of less than 0.05 were accepted as significant (2tailed). As the variables were not normally distributed (Shapiro-Wilk test), non-parametric tests were used. Comparison of repeated measures was made .using Friedman's ANOVA. Post-hoc analysis with the Wilcoxon signedrank test and application of a Bonferroni correction, resulted in significance being set.

\section{Psychological distress}

The studies reviewed in this article used the Symptom Checklist (SCL-90 or SCL-90R) and the Minnesota Multiphasic Personality Inventory (MMPI) to measure general psychological distress. Preoperatively the psychological profiles of patients were generally normal. Despite the wide variation in patients' scores, it has not been reported how many, if any, of the patients had scores outside the normal range. In the studies by Phillips and co-workers, 20\% of patients were distressed preoperatively. Although only $13 \%$ of patients were distressed both before and after surgery, the total number of distressed patients did not change after surgery: some of the patients were distressed only before and some only after surgery [5]. 


\section{Result}

Domain Main results Motivation Several factors motivated patients to have IMF. No single motivation seemed to be common to all patients. Those mentioned most often were improvements in self-confidence, appearance, and function.Psychological distress In general, patients were not distressed and did not suffer from BDD. According to standardized questionnaires, patients were not depressed or anxious.

However, a number of patients reported depression and anxiety Self-esteem and self-confidence Patients' self-esteem did not differ from that of others. After surgery, patients with mid-thirties reported having lower. self-esteem and selfconfidence than before Body image Patients were less satisfied with their facial appearance than others. Satisfaction improved after surgery, but it is not clear whether it reached normal limits Everyday life satisfaction. There were few studies regarding these issues. Patients experienced problems with eating and bullying.

IMF with mid-twenties group reported lower dental trait anxiety (S-DAI) scores than other groups (P = 0.001). S-DAI was significantly associated with frequency of previous traumatic events in 'presurgery' and 'post-surgery' groups $(\mathrm{P}<0.01)$.

\section{Discussion}

The studies on depression and anxiety among patients with intermaxillary fixation tend not to evaluate stress together with depression and anxiety. The studies on intermaxillary fixation stress has been done in history, this is the first study done in central India which includes the psychological stress calculation when under treatment of imf in the period of as 4-6 weeks duration is required. According toIndianperceptive patients develop extra threshold for psychological stress as stress maybe less because of lifestyle.

Initially, psychological burden was measured only in terms of anxiety and depression, and "stress", which focused on difficulty in relaxing, impatience, tension, agitation and irritability, was added later. (2)Our intention was to observe the pattern of stress and anxiety over time. Anxiety was almost stable, but stress increased. Studies have indicated a close affinity between the two, and some of the symptoms of stress such as chronic arousal, tension, fatigue, worry, nervousness, discouragement, shortness of temper, fidgetiness, interrupted sleep, avoidance, and irritability, overlap with those of anxiety [6].

\section{Conclusion}

Throughout the reviewing process, comparisons of the reported results were hampered by the wide variation in both assessment times and applied methods. Furthermore, a number of studies were conducted with small groups and, in some cases, all the necessary statistical information was not reported, or the statistical data and text were incongruent In conclusion, the main findings of this study is during the study of intermaxillary fixation the group of second decade had more stress as compared to third decade cases .

\section{Compliance with ethical standards}

\section{Acknowledgments}

We would like to take the opportunity to thank the RKDF Dental science college for patients, vice chancellor of the institure Dr MC Prashant Pillai for constant support and guidance. Invaluable suggestions and selfless guidance are going to be cherished. Extending warm support by family Mr. Ramakant Sharma and Mrs Mamta Sharma and friends .

\section{Disclosure of conflict of interest}

The authors report no .conflicts of interest. The authors alone are responsible for the content and writing of the paper.

\section{References}

[1] H Sakain. The biochemical study of intermaxillary fixation (IMF) stress in oral surgery inpatients.Kurume Med Journal. 2001; 48(1): 71-7. 
[2] Kumar K, Kumar S, Mehrotra D, Tiwari SC, Kumar V, Khandpur S, Dwivedi RC. Prospective evaluation of psychological burden in patients with oral cancer.Br J Oral Maxillofac Surg. Dec 2018; 56(10): 918-924.

[3] Qin HY, Cheng CW, Tang XD, Bian ZX. Impact of psychological stress on irritable bowel syndrome. World Journal of Gastroenterol. 21 Oct 2014; 20(39).

[4] Daza P, Novy DM, Stanley MA, et al. The depression anxiety stress scale-21: Spanish translation and validation with a Hispanic sample. J PsychopatholBehav Assess. 2002; 24: 195-205.

[5] OME Alanko, et al. Patients' perceptions of orthognathic treatment, well-being, and psychological or psychiatric status: a systematic review. ActaOdontologicaScandinavica. 2010; 68: 249-260.

[6] Bitar AI, Ahmad AI . Anxiety and post-traumatic stress symptoms in orthognathic surgery patients. European Journal of Orthodontics. 2016; 1-6. 